\title{
Hypoconnectivity networks in schizophrenia patients: a voxelwise meta-analysis of rs-fMRI
}

Silvia Ruiz-Torras ( $\square$ silvia.ruiz@ub.edu )

University of Barcelona

Oscar Fernández-Vazquez

University of Barcelona

Cristina Cañete-Massé

University of Barcelona

Maribel Peró-Cebollero

University of Barcelona

Joan Guàrdia-Olmos

University of Barcelona

\section{Research Article}

Keywords: Schizophrenia, fMRI, Resting-State, Functional Connectivity, Meta-analysis

Posted Date: October 19th, 2021

DOl: https://doi.org/10.21203/rs.3.rs-918263/v1

License: (c) (1) This work is licensed under a Creative Commons Attribution 4.0 International License. Read Full License 


\section{Abstract}

In the last few years, the field of brain connectivity has focused on identifying biomarkers to describe different health states and to discriminate between patients and healthy controls through the characterization of brain networks. A particularly interesting case, because of the symptoms' severity, is the work done with samples of patients diagnosed with schizophrenia. This meta-analysis aims to identify connectivity networks with different activation patterns between people diagnosed with schizophrenia and healthy controls. Therefore, we collected primary studies exploring whole brain connectivity by functional magnetic resonance imaging at rest in patients with schizophrenia compared to healthy people. Thus, we identified 25 high-quality studies that included a total of 1285 people with schizophrenia and 1279 healthy controls. The results indicate hypoactivation in the right precentral gyrus and in the left superior temporal gyrus of people with schizophrenia compared with the control group. These regions have been linked to deficits in gesticulation and the experience of auditory hallucinations in people with schizophrenia. A study of heterogeneity demonstrated that the effect size was influenced by the sample size and type of analysis. These results imply new contributions to the knowledge, diagnosis, and treatment of schizophrenia both clinically and in research.

\section{Introduction}

Schizophrenia is the most important severe mental health disorder and implies an extraordinary health problem. Risk factor identification and etiological studies remain unresolved in the scientific response. The genetic and neurobiological factors that have been associated with schizophrenia are quite heterogeneous. Several studies have focused on the dopaminergic dysfunction hypothesis concerning schizophrenia. For example, dopaminergic hypoactivity has been associated with the prefrontal cortex with negative symptomatology (Buckley and Castle, 2015). Currently, there is no biological marker for the diagnosis of schizophrenia. Thus, the early identification of people with a high risk of schizophrenia poses a major public health challenge before they begin to manifest the symptomatology of the disorder (Lewis, 2012). Consequently, a better understanding of this disorder's neurological substrates can help to identify better strategies for early diagnosis and psychological and individualized pharmacological treatment (Nickl-Jockschat and Abel, 2016).

Functional magnetic resonance imaging in the resting state (rs-fMRI) has proven to be a promising tool to contribute to the diagnosis of several disorders, such as autism (Anderson et al., 2011), attention and hyperactivity deficit disorder (Zhu et al., 2008), major depression disorder (Craddock, Holtzheimer, Hu and Mayberg, 2009) and schizophrenia (Chyzhyk and Graña, 2015; Chyzhyk, Savio and Graña, 2015; Rashid et al., 2016; Wang et al., 2018; Qureshi, Oh and Lee, 2019). Thus, it is a noninvasive technique and does not require the active collaboration of the patient (Lee, Smyser, and Shimony, 2013), which is especially important for evaluating brain activity in those populations that have affected their cognitive performance. In the study of connectivity in rest and schizophrenia, evidence has shown significant differences in patients compared to healthy populations. More specifically, the disconnection hypothesis has been studied, a framework in which the diversity of symptoms typical of schizophrenia is 
conceptualized as a result of disconnections in neural networks (Friston and Frith, 1995). In line with this hypothesis, alterations in the default mode network (DMN), the most prominent resting network (Woodward, Rogers and Heckers, 2011), have been shown. In addition, a reduction in precuneus connectivity with other areas was noted in patients with schizophrenia compared to the control group. Connectivity intensity in this area is negatively correlated with the severity of negative symptoms, more specifically with the apathy domain (Forlim et al., 2020). Gangadin et al. (2021) also demonstrated a reduction in the connectivity of the hypocampal-mesencephal-striate network in patients with schizophrenia. In addition, patients showed increased long-range positive connectivity in the right middle frontal gyrus (MFG) and short-range positive connectivity in the right MFG and right superior medial prefrontal cortex, which are brain regions in the anterior DMN (Wang et al., 2018). Hua et al. (2018) noted decreased connectivity between the thalamus and prefrontal cortex and cerebellum but an increase in the connectivity of the thalamus and the motor cortex in patients with schizophrenia. In addition, studies of bilateral asymmetric connectivity have noted that patients with a predominance of positive symptomatology showed significantly more asymmetry to the left hemisphere. Instead, the predominance group of negative symptoms showed more asymmetry to the right. These results suggest that predominantly positive and predominantly negative schizophrenia may have different neural bases and that certain regions in the frontal and temporal lobes, as well as the gyrus and precuneus, play an essential role in mediating the symptoms of this disorder (Ke et al., 2009). Additionally, Chen et al. (2013) showed evidence that cerebellum disconnection is network-specific; that is, the group of patients with schizophrenia showed decreased cerebellum connectivity with the prefrontal lobe and more corticocerebellar connectivity with regions involved in sensory-motor processing, which may be indicative of the deficiencies in inhibition observed in people with schizophrenia. In addition, Li et al. (2019), also with schizophrenia patients, showed reduced insula connectivity with the sensory cortex and putamen compared to people with a high risk of psychotic disorder. Complementarily, schizophrenia patients have shown increased connectivity between the posterior cingulate cortex and the inferior left gyrus, mid-left frontal gyrus, and mid-left temporal gyrus. Conversely, schizophrenia patients have shown decreased connectivity in the executive control network and the dorsal attention network. These results show that resting-state network connectivity is altered in patients with schizophrenia, so the alterations are characterized by reduced segregation between the DMN and the executive control networks in the prefrontal cortex and temporal lobe (Woodward, Rogers and Heckers, 2011). This study found no statistically significant distinctions in the connectivity of the salience network; instead, Huang et al. (2019) showed evidence of hyperconnectivity of the salience network and the prefrontal cortex and cerebellum, as well as hypoconnectivity between the cortico-striatal-thalamic-cortical subcircuit and the salience network.

In recent years, some meta-analyses have explored rs-fMRI in patients with schizophrenia compared to control groups. For example, Xiao et al. (2017) showed evidence that people with schizophrenia had increased connectivity, estimated with regional homogeneity (ReHo), in the right superior frontal and right superior temporal gyrus, as well as decreased ReHo connectivity in the right fusiform gyrus, left superior temporal gyrus, left postcentral gyrus, and right precentral gyrus (focused on ReHo 
studies). Dong et al. (2018) conducted a meta-analysis showing that patients with schizophrenia presented hypoconnectivity in the DMN, affective network (AN), ventral attentional network (VAN), thalamic network (TN), and somatosensory network. They also showed hypoconnectivity between van and TN, VAN and DMN, VAN and the frontoparietal (FN), between FN and TN, and between FN and DMN. Only hyperconnectivity was found between the AN and VAN (focused on seed-based analysis studies). The abovementioned Li et al. (2019) showed evidence through a meta-analysis that supports hypoconnectivity in certain brain networks in schizophrenic patients. More specifically, the self-referential network (superior temporal gyrus) and DMN (right medial prefrontal cortex and left precuneus and anterior cingulate) focused on independent component analysis (ICA) studies. Finally, Gong et al. (2020) showed that people with schizophrenia presented a decreased amplitude of low frequencies (ALFF) in the bilateral postcentral gyrus, bilateral precuneus, left inferior parietal gyrus, and right occipital lobe. In addition, they found an increased ALFF in the right-handed, left inferior frontal gyrus, left inferior temporal gyrus, and right anterior cingulated cortex.

To our knowledge, no meta-analysis includes rs-fMRI studies involving the whole brain, as well as studies that use different analysis techniques (ICA, ReHo, ALFF, falFF, etc.). Moreover, given the incongruences between the studies in this field, the aim of this meta-analysis is to identify functional connectivity networks of the whole brain using a paradigm of rs-fMRI in patients with schizophrenia compared to healthy people (without any neurological or psychiatric disorder). Thus, it is expected that patients diagnosed with schizophrenia will show statistically significant differences in functional connectivity compared to healthy people. In addition, a secondary objective is to analyze the relationship between the effect size and mediator variables, such as sample size, age, gender, etc.

\section{Methods}

Study selection. Two independent investigators performed a bibliographic search using the following databases: PubMed, Web of Science (WoS), Psycinfo, Google Scholar, and Scopus. Additionally, the Boolean algorithm with the keywords used is presented in Supplementary Appendix 1. We included studies published until February 28, 2021. This meta-analysis was conducted according to the "Preferred Reporting Items for Systematic Reviews and Meta-analysis (PRISMA)" guidelines. The inclusion criteria for the studies were as follows: 1) they were published in English or Spanish; 2) the full text was available; 3 ) they were a primary study in a human population; 4) they included a patient group diagnosed with schizophrenia following the DSM-IV criteria or the structured clinical interview for DSM-IV (SCID); 5) they compared brain activation between schizophrenia patients and healthy people; 6 ) they used rs-fMRI; or 7) the studies reported Montreal Neurological Institute (MNI) or Talairach coordinates of the whole brain contrast comparing schizophrenia persons and control subjects. The exclusion criteria were as follows: 1) systematic reviews or meta-analysis; 2) methodologic studies; 3) patients with schizophrenia with other psychiatric or neurological disorders; or 4) studies focused on dynamic connectivity or use graph analysis or any other technique that does not identify coordinates. The studies were screened out as shown in Figure 1. Our search yielded a total of 3563 studies [WoS $(n=1123)$, Scopus ( $n=823)$, PubMed $(n=729)$, Psyclnfo $(n=543)$, and Google Scholar $(n=345)]$. Subsequently, 
2106 duplicate papers were removed through Mendeley, and 64 duplicate papers were removed via Rayyan. A total of 1234 studies were excluded after title/abstract screening because they did not meet the inclusion criteria. Later, 159 articles were sought to read the full text, but 8 studies could not be found. Thus, after the full-text screening, 73 studies were excluded because they did not provide information about the peak activation coordinates, 16 because they did not report on the statistics associated with the coordinates, and 37 because they did not analyze the whole brain. Finally, only 25 studies matching our inclusion criteria were included and are marked with an * in the reference list. In addition, we obtained a $100 \%$ rate of agreement between the two investigators for the study search and selection.

Voxel-Wise Meta-analysis. We used seed-based $d$ mapping (SDM) software (available at http://www.sdmproject.com) to analyze the differences between schizophrenia patients and healthy subjects. The approach details have been described in Radua and Mataix (2012) or Müller et al. (2018). First, the reported peak coordinates of all functional differences, which were statistically significant at the whole brain level in these studies, were chosen. We ensured that all included studies used the same statistical threshold throughout the whole brain to avoid possible bias toward regions with liberal thresholds. Thus, we considered the minimum threshold to be defined by a .001 significance value and Student's $t$ reference value with the degrees of freedom of each study estimated by the conventional expression $\left(n_{1}+n_{2}-2\right)$. Second, we recreated peak coordinates for each study with a standard MNI map of the group difference effect size based on their peak $t$ value by means of a nonnormalized Gaussian kernel to the voxels near the peak, which assigns higher values to the voxels closer to peaks. Third, the mean map was obtained by voxelwise calculation of the study map random-effects mean, weighted by the sample size. Fourth, to correctly balance the sensitivity and specificity, we used the $p$ value of 0.05 as the main threshold with an additional peak height of $z=1$. Jackknife sensitivity analysis was performed to test the replicability of the results. After the calculation of Cohen's $d$ and the confidence interval (Cl) analysis of the different papers was performed, a descriptive analysis of every paper result was resumed in different images to clarify the results obtained in every included study.

Quality Assessment. We assessed the quality of the included studies using a checklist consisting of 11 items that focused on the clinical characteristics of the participants, the neuroimaging and data analysis methodology, the results, and the conclusions of the studies. The quality assessment scale is shown in Supplementary Appendix 2. This checklist was based on previous metanalyses and has been described elsewhere (Shepherd, Matheson, Laurens and Green, 2012; Chen et al., 2015). One author reviewed the included studies and determined a complete rating. The resulting scores were discussed between two investigators, and a consensus quality score was obtained.

\section{Results}

Studies included in the meta-analysis. Supplementary Appendix number 3 shows the data obtained in each study to describe each mediator variable for each analyzed paper. Table 1 shows the basic 
descriptive statistics of the mediator variables. We want to highlight that the total number of patients analyzed assumes a significant number $(n=1285)$ and a control group $(n=1279)$. With regard to the techniques used to estimate connectivity networks, the most common are ALFF (20.68\%) and ReHo (34.48\%).

Meta-analysis Result. In Figures 2 and 3, the forest plot shows the effect size of each study, as well as the total mean of the effect size and a confidence interval of $95 \%$. Conversely, if the effect size was negative, the patient group showed decreased activation compared to the healthy group. Therefore, we can see that the most significant positive effect size found is 1.715 , with the upper limit being 2.448 . On the other hand, the highest negative effect size is -1.673 , with the lower limit being -2.361 . Notably, in the case of negative differences, the work of Turner et al. (2013) and Fryer et al. (2015) show a lower width of the confidence interval, and the same work by Turner et al. (2013) shows the most accurate interval in the case of positive differences.

In this meta-analysis, people with schizophrenia did not show any hyperactivation compared to controls. However, they show decreased activation in the right precentral gyrus, specifically in the Brodmann area (BA) 4. In addition, they also show hypoactivation in the left superior temporal gyrus corresponding to BA 22. Thus, two clusters were found, one with 640 voxels and one with 150 voxels. The results are displayed in Table 2.

In addition, Figure 4 shows the graphical representation of the areas that are hypoactivated (visualized with BrainNet Viewer; Xia, Wang and He, 2013; http://www.nitrc.org/projects/bnv/). Note that the size of the node is proportional to the voxels it represents. That is, the larger the node, the more voxels there are in that area and the larger the region it represents. It is also worth noting that the nodes represented in blue correspond to the right precentral gyrus (BA 4), and the yellow nodes refer to the left superior temporal gyrus (BA 22).

Reliability analysis. A jackknife sensitivity analysis (Table 3 ) was carried out to check the replicability of the results. This analysis revealed that the right precentral gyrus (with coordinates of $50,-10,40$ ) was replicable in all 29/29 datasets (each dataset with one study left out) and that the left superior temporal gyrus $(-58,-20,4)$ was replicable in $26 / 29$ datasets (the results were not confirmed only in 3 of the simulations performed, that is, $89.66 \%$ reliability). Therefore, we can establish a very high reliability of the results obtained. 
Publication bias analysis. In Figures 5 and 6 , we can see funnel plots wherein publication bias is graphically displayed. As we can see, the graphs suggest that there is no publication bias since the points (which correspond to the effect sizes of each study) are distributed uniformly on one side and on the other side of the value 0 of the abscises axis (effect size). Therefore, in our case, we can see how studies have been published with increasingly significant effects. In addition, Table 4 shows the values of $Z$ statistics, as well as their significance $(p=.504$ and $p=.751)$, which indicates that there is no bias.

Heterogeneity analysis. To determine the possible heterogeneity between the studies included in the present meta-analysis, $Q$ and $P^{2}$ statistics were estimated. The results obtained are in (Table 5), where the values of tau $(\tau)$ representing the variance of the effect size distribution are displayed. In addition, we can see that $\mathrm{Q}$ statistics, from both positive and negative peaks, are statistically significant $(p<.001)$. In this way, there is heterogeneity between the different studies, so it is appropriate to explore the mediator variables that could explain this phenomenon. On the other hand, it must be pointed out that the degree of heterogeneity is calculated by the $I^{2}$ index, as we can see are values that indicate a moderate degree of heterogeneity.

As mentioned above, the fact that there is heterogeneity between the studies included in the metaanalysis leads us to perform an analysis of possible mediator variables that could explain the variability between the effect sizes (here considered in absolute value). Since more than one statistically significant effect is present in some works, the effect size of each article analyzed is estimated by the mean of the effect size included in each paper. The following variables were explored: type of data analysis used in studies (ReHo, ALFF, fALFF, etc.), sample size, age, and sex of the patient group and control group, total, general, positive, and negative PANSS scores applied to the group of patients with schizophrenia, illness duration, and quality of the studies analyzed.

Categorical variables. First, Welch's t-test was used to analyze the relationship between the effect size and the type of analysis (ReHo or ALFF, the rest of the techniques were underrepresented and were eliminated from this analysis). The results indicate that there was a statistically significant relationship between the effect size and the type of analysis used $\left(t=-2.381 ; d f=13.927 ; p_{\text {uni }}=.016 ; r=.538\right)$. In fact, the mean effect size in ReHo is $d=1.242$, and in ALFF studies, $d=.964$, which suggests that studies using ReHo can obtain a greater effect size than those using ALFF, and this effect has high intensity according to Cohen's criteria. 
Quantitative variables: Meta-regression analysis. A meta-regression was performed to analyze whether the quantitative variables described above (Table 1) had a statistically significant impact. Thus, Table 6 presents the minimum quadratic estimates of each meta-regression to evaluate the effect of each mediator variable on the estimate of the effect size.

From the above table, it follows that only sample sizes are statistically significant predictors of the effect size heterogeneity. The negative value of the regression coefficients indicates that works with higher sample sizes obtain lower values of the effect. In summary, the analysis performed with mediator variables indicates that, in the studies analyzed, the effect size is higher in ReHo estimated with smaller samples.

\section{Discussion}

To our knowledge, this is the first meta-analysis to study rs-fMRI in the whole brain in patients with schizophrenia compared to healthy people, including studies that use different analysis techniques. The results indicate that there was hypoactivation of the right precentral gyrus and left superior temporal gyrus in patients with schizophrenia compared to the control groups.

These results are congruent with other findings. Dong et al. (2018) also showed evidence in his metaanalysis (only ALFF studies) of a reduction in connectivity in the left superior temporal gyrus in patients with schizophrenia. Similarly, another meta-analysis also showed evidence of decreased ReHo in this area (Xiao et al., 2017). Additionally, in a systematic review, connectivity alterations were found in this area in studies performed with both rs-fMRI and task fMRI (Mwansisya et al., 2017). In addition, children of schizophrenic patients also show reduced activation of the left superior temporal gyrus during hearing comprehension (Rajarethinam et al., 2011). It should be noted that dysfunction in this region has been related to the presence of auditory hallucinations in patients with schizophrenia (Carter et al., 1994; Hugdahl, Løberg and Nygård, 2009). More specifically, Plaze et al. (2006) showed evidence that the anterior area of the left superior temporal gyrus is part of the brain network associated with the perception of auditory hallucinations in patients with schizophrenia, indicating that activity in this cortical region may be related to the severity of hallucinations. Activation of this area has also been demonstrated during the experience of auditory verbal hallucinations (Sommer et al., 2012). Consistent with our results, Gong et al. (2020) showed evidence of ALFF alteration in the right precentral gyrus. In addition, Li et al. (2019) found hypoconnectivity between the right precentral gyrus, which is involved in motor function, and the postcentral and precentral gyrus and cerebellum. Additionally, Xiao et al. (2017) showed evidence of decreased ReHo in the right central gyrus. In addition, hypoactivation in this area has been shown in relatives of people with schizophrenia compared to the control group (Scognamiglio and Houenou, 2014). It should be noted that dysfunctions in praxis networks in patients with schizophrenia, which includes the right precentral gyrus, correlate with deficits in the gesticulation of these patients (Wüthrich et al., 2020). 
Our results are congruent with the meta-analysis of Gong et al. (2020), and we found that the duration of illness was not related to the effect size. Nevertheless, unlike the meta-analysis of Gong et al. (2020), we did not find that any of the PANSS scores were related to the effect size.

There are some limitations in this meta-analysis. First, we did not include those studies that did not report the coordinates or those that did not report the associated statistics. In addition, we have not taken into account the different subtypes of schizophrenia, which would be interesting for future research.

Additionally, some analysis techniques used by the studies that we included were underrepresented and could not be taken into account when evaluating whether they could predict the effect size. There were several missing values regarding PANSS scores.

Finally, concerning the strengths of this work, it must be noted that a thorough search of articles by two independent researchers was carried out following the recommendations of PRISMA. In addition, no publication bias has been found. An analysis of the studies' quality was carried out, which is high. Just as the results obtained are highly reliable. They also support other findings, and in addition, new scientific and clinical contributions have been made to the knowledge, diagnosis and treatment of schizophrenia

\section{References}

Aleman, A., Kahn, R. S. \& Selten, J. P. (2003). Sex differences in the risk of schizophrenia: evidence from meta-analysis. Archives of general psychiatry, 60(6), 565-571. https://doi: 10.1001/archpsyc.60.6.565

* Alonso-Solís, A., Vives-Gilabert, Y., Portella, M. J., Rabella, M., Grasa, E. M., Roldán, A., Keymer-Gausset, A., Molins, C., Núñez-Marín, F., Gómez-Ansón, B., Álvarez, E. \& Corripio, I. (2017). Altered amplitude of low frequency fluctuations in schizophrenia patients with persistent auditory verbal hallucinations.

Schizophrenia research, 189, 97-103. https://doi: 10.1016/j.schres.2017.01.042

Anderson, J. S., Nielsen, J. A., Froehlich, A. L., DuBray, M. B., Druzgal, T. J., Cariello, A. N. \& Lainhart, J. E. (2011). Functional connectivity magnetic resonance imaging classification of autism. Brain, 134(12), 3742-3754. https://doi: 10.1093/brain/awr263

* Bai, Y., Wang, W., Xu, J., Zhang, F., Yu, H., Luo, C., Wang, L., Chen, X., Shan, B., Xu, L., Xu, X. \& Cheng, Y. (2016). Altered resting-state regional homogeneity after 13 weeks of paliperidone injection treatment in schizophrenia patients. Psychiatry Research: Neuroimaging, 258, 37-43. https://doi:

10.1016/j.pscychresns.2016.10.008

Buckley, P. F. \& Castle, D. J. (2015). Schizophrenia (Oxford Psychiatry Library). Oxford University Press. https://doi: 10.1093/med/9780198712831.001.0001

Carter, C. S., Robertson, L. C., Nordahl, T. E., Chaderjian, M. \& O'Shora-Celaya, L. (1994). Left superior temporal gyrus dysfunction and auditory hallucinations in schizophrenia: A cognitive neuropsychological study. Biological Psychiatry, 35(9), 659. 
Chen, Y. L., Tu, P. C., Lee, Y. C., Chen, Y. S., Li, C. T. \& Su, T. P. (2013). Resting-state fMRI mapping of cerebellar functional dysconnections involving multiple large-scale networks in patients with schizophrenia. Schizophrenia research, 149(1-3), 26-34. https://doi: 10.1016/j.schres.2013.05.029

Chen, Z., Q., Du, M. Y., Zhao, Y. J., Huang, X. Q., Li, J., Lui, S., Hu, J., Sun, H., Liu, J., Kemp, G. J. \& Gong, Q. $\mathrm{Y}$. (2015). Voxel-wise meta-analyses of brain blood flow and local synchrony abnormalities in medicationfree patients with major depressive disorder. Journal of Psychiatry \& Neuroscience. 40, 401-411. https://doi: 10.1503/jpn.140119

Chyzhyk, D. \& Graña, M. (2015). Classification of schizophrenia patients on lattice computing restingstate fMRI features. Neurocomputing, 151, 151-160. https://doi.org/10.1016/j.neucom.2014.09.075

Chyzhyk, D., Savio, A. \& Graña, M. (2015). Computer aided diagnosis of schizophrenia on resting state fMRI data by ensembles of ELM. Neural Networks, 68, 23-33. https://doi: 10.1016/j.neunet.2015.04.002

Craddock, R. C., Holtzheimer III, P. E., Hu, X. P. \& Mayberg, H. S. (2009). Disease state prediction from resting state functional connectivity. Magnetic Resonance in Medicine: An Official Journal of the International Society for Magnetic Resonance in Medicine, 62(6), 1619-1628. https://doi:

10.1002/mrm.22159

De Marco, M., Meneghello, F., Duzzi, D., Rigon, J., Pilosio, C. \& Venneri, A. (2016). Cognitive stimulation of the default-mode network modulates functional connectivity in healthy aging. Brain research bulletin, 121, 26-41. https://doi: 10.1016/j.brainresbull.2015.12.001

* Ding, Y., Ou, Y., Su, Q., Pan, P., Shan, X., Chen, J., Liu, F., Zhang, Z., Zhao, J. \& Guo, W. (2019). Enhanced global-brain functional connectivity in the left superior frontal gyrus as a possible endophenotype for schizophrenia. Frontiers in neuroscience, 13, 145. https://doi.org/10.3389/fnins.2019.00145

Dong, D., Wang, Y., Chang, X., Luo, C. \& Yao, D. (2018). Dysfunction of large-scale brain networks in schizophrenia: a meta-analysis of resting-state functional connectivity. Schizophrenia bulletin, 44(1), 168181. https://doi: 10.1093/schbul/sbx034

Friston, K. J. (2011). Functional and effective connectivity: a review. Brain connectivity, 1(1), 13-36. https://doi: 10.1089/brain.2011.0008

Friston, K. J. \& Frith, C. D. (1995). Schizophrenia: a disconnection syndrome. Clin Neurosci, 3(2), 89-97.

Forlim, C. G., Klock, L., Bächle, J., Stoll, L., Giemsa, P., Fuchs, M., Shoofs, N., Montag, C., Gallinat, J. \& Kühn, S. (2020). Reduced Resting-State connectivity in the precuneus is correlated with Apathy in patients with Schizophrenia. Scientific reports, 10(1), 1-8. https://doi: 10.1038/s41598-020-59393-6

* Fryer, S. L., Roach, B. J., Ford, J. M., Turner, J. A., Van Erp, T. G., Voyvodic, J., Preda, A., Belger, A., Bustillo, J., O'Leary, D., Mueller, B. A., Lim, K. O., McEwen, S. C., Calhoun, V. D., Diaz, M., Glover, G., Greve, D., Wible, C. G., Vaidya, J., Potkin, S. G. \& Mathalon, D. H. (2015). Relating intrinsic low-frequency BOLD cortical 
oscillations to cognition in schizophrenia. Neuropsychopharmacology, 40(12), 2705-2714. https://doi.org/10.1038/npp.2015.119

Gangadin, S. S., Cahn, W., Scheewe, T. W., Pol, H. E. H., \& Bossong, M. G. (2021). Reduced resting state functional connectivity in the hippocampus-midbrain-striatum network of schizophrenia patients. Journal of psychiatric research, 138, 83-88. https://doi.org/10.1016/j.jpsychires.2021.03.041

* Gao, B., Wang, Y., Liu, W., Chen, Z., Zhou, H., Yang, J., Cohen, Z., Zhu, Y. \& Zang, Y. (2015). Spontaneous activity associated with delusions of schizophrenia in the left medial superior frontal gyrus: a restingstate fMRI study. PLoS One, 10(7), e0133766. https://doi: 10.1371/journal.pone.0133766

* Gao, S., Lu, S., Shi, X., Ming, Y., Xiao, C., Sun, J., Yao, H. \& Xu, X. (2018). Distinguishing between treatment-resistant and non-treatment-resistant schizophrenia using regional homogeneity. Frontiers in psychiatry, 9, 282. https://doi: 10.3389/fpsyt.2018.00282

Gong, J., Wang, J., Luo, X., Chen, G., Huang, H., Huang, R., Huang, L. \& Wang, Y. (2020). Abnormalities of intrinsic regional brain activity in first-episode and chronic schizophrenia: a meta-analysis of resting-state functional MRI. Journal of Psychiatry \& Neuroscience.

* Gou, N., Liu, Z., Palaniyappan, L., Li, M., Pan, Y., Chen, X., Tao, H., Wu, G., Ouyang, X., Wang, Z., Dou, T., Xue, Z. \& Pu, W. (2018). Effects of DISC1 polymorphisms on Resting-State Spontaneous Neuronal Activity in the early-stage of schizophrenia. Frontiers in psychiatry, 9, 137.

https://doi.org/10.3389/fpsyt.2018.00137

* Guo, W., Liu, F., Chen, J., Wu, R., Li, L., Zhang, Z., Chen, H. \& Zhao, J. (2017). Treatment effects of olanzapine on homotopic connectivity in drug-free schizophrenia at rest. The World Journal of Biological Psychiatry, 19(sup3), S106-S114. https://doi: 10.1080/15622975.2017.1346280

* Guo, W., Liu, F., Chen, J., Wu, R., Li, L., Zhang, Z. \& Zhao, J. (2016). Olanzapine modulation of long-and short-range functional connectivity in the resting brain in a sample of patients with schizophrenia. European Neuropsychopharmacology, 27(1), 48-58. https://doi: 10.1016/j.euroneuro.2016.11.002

* Guo, W., Liu, F., Xiao, C., Liu, J., Yu, M., Zhang, Z., Zhang, J. \& Zhao, J. (2015). Increased short-range and long-range functional connectivity in first-episode, medication-naive schizophrenia at rest. Schizophrenia research, 166(1-3), 144-150. https://doi: 10.1016/j.schres.2015.04.034

Hua, J., Blair, N. I., Paez, A., Choe, A., Barber, A. D., Brandt, A. \& Margolis, R. L. (2019). Altered functional connectivity between sub-regions in the thalamus and cortex in schizophrenia patients measured by resting state BOLD fMRI at 7T. Schizophrenia research, 206, 370-377. https://doi:

10.1016/j.schres.2018.10.016

Huang, H., Botao, Z., Jiang, Y., Tang, Y., Zhang, T., Tang, X. \& Wang, J. (2019). Aberrant resting-state functional connectivity of salience network in first-episode schizophrenia. Brain imaging and behavior, 1- 
Hugdahl, K., Løberg, E. M. \& Nygård, M. (2009). Left temporal lobe structural and functional abnormality underlying auditory hallucinations. Frontiers in neuroscience, $3,1$.

https://doi.org/10.3389/neuro.01.001.2009

Kahn, R. S., Sommer, I. E., Murray, R. M., Lindenberg, A., Weinberg, D. R. \& Cannon, T. D. (2015).

Schizophrenia. Nat Rev Dis Primers, 1, 15067. https://doi: 10.1038/nrdp.2015.67

Ke, M., Zou, R., Shen, H., Huang, X., Zhou, Z., Liu, Z. \& Hu, D. (2010). Bilateral functional asymmetry disparity in positive and negative schizophrenia revealed by resting-state fMRI. Psychiatry Research: Neuroimaging, 182(1), 30-39. https://doi: 10.1016/j.pscychresns.2009.11.004

Lee, M. H., Smyser, C. D. \& Shimony, J. S. (2013). Resting-state fMRI: a review of methods and clinical applications. American Journal of neuroradiology, 34(10), 1866-1872. https:// doi: 10.3174/ajnr.A3263

Lewis, D. A. (2012). Cortical circuit dysfunction and cognitive deficits in schizophrenia-implications for preemptive interventions. European Journal of Neuroscience, 35(12), 1871-1878. https:// doi:

10.1111/j.1460-9568.2012.08156

* Li, H., Ou, Y., Liu, F., Chen, J., Zhao, J., Guo, W. \& Fan, X. (2019). Reduced connectivity in anterior cingulate cortex as an early predictor for treatment response in drug-naive, first-episode schizophrenia: a global-brain functional connectivity analysis. Schizophrenia research, 215, 337-343. https:// doi: 10.1016/j.schres.2019.09.003

Li, S., Hu, N., Zhang, W., Tao, B., Dai, J., Gong, Y., Tan, Y., Cai, D. \& Lui, S. (2019). Dysconnectivity of multiple brain networks in schizophrenia: a meta-analysis of resting-state functional connectivity. Frontiers in psychiatry, 10, 482. https://doi.org/10.3389/fpsyt.2019.00482

* Li, Z., Lei, W., Deng, W., Zheng, Z., Li, M., Ma, X., Wang, Q., Huang, X., Li, N., Collier, D. A., Gong, Q. \& Li, T. (2017). Aberrant spontaneous neural activity and correlation with evoked-brain potentials in first-episode, treatment-naïve patients with deficit and non-deficit schizophrenia. Psychiatry Research: Neuroimaging, 261, 9-19. https://doi.org/10.1016/j.pscychresns.2017.01.001

* Liang, Y., Shao, R., Zhang, Z., Li, X., Zhou, L. \& Guo, S. (2019). Amplitude of low-frequency fluctuations in childhood-onset schizophrenia with or without obsessive-compulsive symptoms: a resting-state functional magnetic resonance imaging study. Archives of medical science: AMS, 15(1), 126. https://doi: 10.5114/aoms.2018.73422

* Liao, W., Yang, S., Li, J., Fan, Y. S., Duan, X., Cui, Q. \& Chen, H. (2019). Nicotine in action: cigarette smoking modulated homotopic functional connectivity in schizophrenia. Brain imaging and behavior, 13(6), 1612-1623. https://doi: 10.1007/s11682-018-0001-3 
* Liu, C., Xue, Z., Palaniyappan, L., Zhou, L., Liu, H., Qi, C., Wu, G., Mwansisya, T. E., Tao, H., Chen, X., Huang, X., Liu, Z. \& Pu, W. (2016). Abnormally increased and incoherent resting-state activity is shared between patients with schizophrenia and their unaffected siblings. Schizophrenia research, 171(1-3), 158165. https://doi.org/10.1016/j.schres.2016.01.022

* Liu, H., Liu, Z., Liang, M., Hao, Y., Tan, L., Kuang, F., Yi, Y., Xu, L. \& Jiang, T. (2006). Decreased regional homogeneity in schizophrenia: a resting state functional magnetic resonance imaging study. Neuroreport, 17(1), 19-22. https://doi: 10.1097/01.wnr.0000195666.22714.35

* Liu, Y., Zhang, Y., Lv, L., Wu, R., Zhao, J. \& Guo, W. (2017). Abnormal neural activity as a potential biomarker for drug-naive first-episode adolescent-onset schizophrenia with coherence regional homogeneity and support vector machine analyses. Schizophrenia research, 192, 408-415. https://doi: 10.1016/j.schres.2017.04.028

Lv, H., Wang, Z., Tong, E., Williams, L. M., Zaharchuk, G., Zeineh, M. \& Wintermark, M. (2018). Resting-state functional MRI: everything that nonexperts have always wanted to know. American Journal of Neuroradiology, 39(8), 1390-1399. https://doi: 10.3174/ajnr.A5527

Mash, L. E., Linke, A. C., Olson, L. A., Fishman, I., Liu, T. T. \& Müller, R. A. (2019). Transient states of network connectivity are atypical in autism: A dynamic functional connectivity study. Human brain mapping, 40(8), 2377-2389. https://doi: 10.1002/hbm.24529

Müller, V. I., Cieslik, E. C., Laird, A. R., Fox, P. T., Radua, J., Mataix-Cols, D. \& Eickhoff, S. B. (2018). Ten simple rules for neuroimaging meta-analysis. Neuroscience \& Biobehavioural Reviews, 84, 151161. https://doi: 10.1016/j.neubiorev.2017.11.012

Mwansisya, T. E., Hu, A., Li, Y., Chen, X., Wu, G., Huang, X. \& Liu, Z. (2017). Task and resting-state fMRI studies in first-episode schizophrenia: A systematic review. Schizophrenia research, 189, 9-18. https://doi: 10.1016/j.schres.2017.02.026

Nickl-Jockschat, T. \& Abel, T. (2016). Historical and Clinical Overview: Implications for Schizophrenia Research. The Neurobiology of Schizophrenia, 3-13. https://doi.org/10.1016/B978-0-12-801829-3.000094

Page, M. J., McKenzie, J. E., Bossuyt, P. M., Boutron, I., Hoffmann, T. C., Mulrow, C. D., Shamseer, L., Tetzlaff, J. M., Akl, E. A., Brennan, S. E., Chou, R., Glanville, J., Grimshaw, J. M., Hróbjartsson, A., Lalu, M. M., Li, T., Loder, E. W., Mayo-Wilson, E., McDonald, S. \& Moher, D. (2021). The PRISMA 2020 statement: an updated guideline for reporting systematic reviews. BMJ, 372. https://doi.org/10.1136/bmj.n71

Plaze, M., Bartrés-Faz, D., Martinot, J. L., Januel, D., Bellivier, F., De Beaurepaire, R. \& Paillère-Martinot, M. L. (2006). Left superior temporal gyrus activation during sentence perception negatively correlates with auditory hallucination severity in schizophrenia patients. Schizophrenia research, 87(1-3), 109-115. https://doi: 10.1016/j.schres.2006.05.005 
Poldrack, R. A., Mumford, J. A. \& Nichols, T. E. (2011). Handbook of functional MRI data analysis. Cambridge University Press.

Qureshi, M. N. I., Oh, J. \& Lee, B. (2019). 3D-CNN based discrimination of schizophrenia using restingstate fMRI. Artificial intelligence in medicine, 98, 10-17. https://doi.org/10.1016/j.artmed.2019.06.003

Radua, J. \& Mataix, D. (2012). Meta-analytic methods for neuroimaging data explained. Biological Mood Anxiety Disorders, 2, 6. https://doi: 10.1186/2045-5380-2-6

Rajarethinam, R., Venkatesh, B. K., Peethala, R., Phan, K. L. \& Keshavan, M. (2011). Reduced activation of superior temporal gyrus during auditory comprehension in young offspring of patients with schizophrenia. Schizophrenia research, 130(1-3), 101-105. https://doi: 10.1016/j.schres.2011.05.025

Rashid, B., Arbabshirani, M. R., Damaraju, E., Cetin, M. S., Miller, R., Pearlson, G. D. \& Calhoun, V. D. (2016). Classification of schizophrenia and bipolar patients using static and dynamic resting-state fMRI brain connectivity. Neuroimage, 134, 645-657. https://doi: 10.1016/j.neuroimage.2016.04.051

* Ren, W., Lui, S., Deng, W., Li, F., Li, M., Huang, X., Wang, Y., Li, T., Sweeney, J. A. \& Gong, Q. (2013). Anatomical and functional brain abnormalities in drug-naive first-episode schizophrenia. American Journal of Psychiatry, 170(11), 1308-1316. https://doi: 10.1176/appi.ajp.2013.12091148

Scognamiglio, C. \& Houenou, J. (2014). A meta-analysis of fMRI studies in healthy relatives of patients with schizophrenia. Australian \& New Zealand Journal of Psychiatry, 48(10), 907-916. https://doi: $10.1177 / 0004867414540753$

Shepherd, A. M., Matheson, S. L. , Laurens, K. R. \& Green, M. J. (2012). Systematic meta- analysis of insula volume in schizophrenia. Biological Psychiatry, 72, 775-84. https://doi:

10.1016/j.biopsych.2012.04.020

Smitha, K. A., Akhil Raja, K., Arun, K. M., Rajesh, P. G., Thomas, B., Kapilamoorthy, T. R. \& Kesavadas, C. (2017). Resting state fMRI: A review on methods in resting state connectivity analysis and resting state networks. The neuroradiology journal, 30(4), 305-317. https://doi: 10.1177/1971400917697342

Sommer, I. E., Clos, M., Meijering, A. L., Diederen, K. M. \& Eickhoff, S. B. (2012). Resting state functional connectivity in patients with chronic hallucinations. PloS one, 7(9), e43516. https://doi:

10.1371/journal.pone.0043516

* Turner, J. A., Damaraju, E., Van Erp, T. G., Mathalon, D. H., Ford, J. M., Voyvodic, J., Mueller, B. A., Belger, A., Bustillo, J., McEwen, S., Potkin, S. G. \& Calhoun, V. D. (2013). A multi-site resting state fMRI study on the amplitude of low frequency fluctuations in schizophrenia. Frontiers in neuroscience, 7, 137. https://doi: 10.3389/fnins.2013.00137

* Wang, S., Zhan, Y., Zhang, Y., Lyu, L., Lyu, H., Wang, G., Wu, R., Zhao, J. \& Guo, W. (2018). Abnormal longand short-range functional connectivity in adolescent-onset schizophrenia patients: a resting-state fMRI 
study. Progress in Neuro-Psychopharmacology and Biological Psychiatry, 81, 445-451. https://doi: 10.1016/j.pnpbp.2017.08.012

Wang, S., Zhang, Y., Lv, L., Wu, R., Fan, X., Zhao, J. \& Guo, W. (2018). Abnormal regional homogeneity as a potential imaging biomarker for adolescent-onset schizophrenia: a resting-state fMRI study and support vector machine analysis. Schizophrenia research, 192, 179-184. https://doi:

10.1016/j.schres.2017.05.038

White, T. \& Calhoun, V. D. (2019). Dissecting static and dynamic functional connectivity: Example from the autism spectrum. Journal of experimental neuroscience, 13, 1179069519851809. https://doi: $10.1177 / 1179069519851809$

Woodward, N. D., Rogers, B. \& Heckers, S. (2011). Functional resting-state networks are differentially affected in schizophrenia. Schizophrenia research, 130(1-3), 86-93. https://doi:

10.1016/j.schres.2011.03.010

Wüthrich, F., Viher, P. V., Stegmayer, K., Federspiel, A., Bohlhalter, S., Vanbellingen, T. \& Walther, S. (2020). Dysbalanced resting-state functional connectivity within the praxis network is linked to gesture deficits in schizophrenia. Schizophrenia bulletin, 46(4), 905-915. https://doi: 10.1093/schbul/sbaa008

Xia M., Wang J. \& He Y. (2013). BrainNet Viewer: a network visualization tool for human brain connectomics. PloS one; 8

Xiao, B., Wang, S., Liu, J., Meng, T., He, Y. \& Luo, X. (2017). Abnormalities of localized connectivity in schizophrenia patients and their unaffected relatives: a meta-analysis of resting-state functional magnetic resonance imaging studies. Neuropsychiatric disease and treatment, 13, 467. https://doi: $10.2147 /$ NDT.S126678

* Yan, W., Zhang, R., Zhou, M., Lu, S., Li, W., Xie, S. \& Zhang, N. (2020). Relationships between abnormal neural activities and cognitive impairments in patients with drug-naive first-episode schizophrenia. $B M C$ psychiatry, 20, 1-9. https://doi: 10.1186/s12888-020-02692

* Yu, R., Hsieh, M. H., Wang, H. L. S., Liu, C. M., Liu, C. C., Hwang, T. J., Chien, Y. L., Hwu, H. G. \& Tseng, W. Y. I. (2013). Frequency dependent alterations in regional homogeneity of baseline brain activity in schizophrenia. PloS one, 8(3), e57516. https://doi: 10.1371/journal.pone.0057516

* Zhao, X., Yao, J., Lv, Y., Zhang, X., Han, C., Chen, L., Ren, F., Jin, Z., Li, Y. \& Sui, Y. (2019). Abnormalities of regional homogeneity and its correlation with clinical symptoms in Naïve patients with first-episode schizophrenia. Brain imaging and behavior, 13(2), 503-513. https://doi: 10.1007/s11682-018-9882-4

* Zheng, J., Zhang, Y., Guo, X., Duan, X., Zhang, J., Zhao, J. \& Chen, H. (2016). Disrupted amplitude of lowfrequency fluctuations in antipsychotic-naive adolescents with early-onset schizophrenia. Psychiatry Research: Neuroimaging, 249, 20-26. https://doi: 10.1016/j.pscychresns.2015.11.006 
Zhu, C. Z., Zang, Y. F., Cao, Q. J., Yan, C. G., He, Y., Jiang, T. Z. \& Wang, Y. F. (2008). Fisher discriminative analysis of resting-state brain function for attention-deficit/hyperactivity disorder. Neuroimage, 40(1), 110-120. https://doi: 10.1016/j.neuroimage.2007.11.029

* Zhu, F., Liu, F., Guo, W., Chen, J., Su, Q., Zhang, Z., Li, H., Fan, X. \& Zhao, J. (2018). Disrupted asymmetry of inter-and intra-hemispheric functional connectivity in patients with drug-naive, first-episode schizophrenia and their unaffected siblings. EBioMedicine, 36,429-

435.https://doi.org/10.1016/j.ebiom.2018.09.012

\section{Tables}

Table 1. Descriptive statistics of the observed distributions of mediator quantitative variables.

\begin{tabular}{|c|c|c|c|c|}
\hline Mediator variables & Minimum & Maximum & Mean & Standard deviation \\
\hline Illness duration & .446 & 16.948 & 4.003 & 4.980 \\
\hline Mean age schizophrenia group & 13 & 40.050 & 26.650 & 7.732 \\
\hline Mean age control group & 12.900 & 37.800 & 26.839 & 7.493 \\
\hline$\%$ men schizophrenia group & 30.610 & 82.350 & 56.035 & 12.442 \\
\hline$\%$ men control group & 39.540 & 82.350 & 56.204 & 11.285 \\
\hline Sample size schizophrenia group & 14 & 168 & 44.31 & 37.179 \\
\hline Sample size control group & 14 & 166 & 44.10 & 37.527 \\
\hline PANSS Total & 19.240 & 49.120 & 38.019 & 7.899 \\
\hline PANSS Negative & 8.410 & 27.490 & 19.601 & 4.200 \\
\hline PANSS Positive & 9.530 & 27.530 & 19.297 & 4.801 \\
\hline PANSS General & 37.290 & 102.000 & 79.457 & 15.269 \\
\hline Quality of the studies & 9.50 & 11.00 & 10.760 & .385 \\
\hline
\end{tabular}

Table 2. Major differences in activation between patients with schizophrenia and the control group in the present meta-analysis. 


Region $\frac{\text { MNI coordinates }}{\mathrm{x} \text { y } \mathrm{z}}$ score p value Voxels Brodmann's area

$\mathrm{SQZ}<\mathrm{HC}$

Right precentral gyrus

$50 \quad-10 \quad 40$

40

$-5.902$

.009

640

BA 4

Left superior temporal gyrus

$\begin{array}{lllllll}-58 & -20 & 4 & -6.128 & .009 & 150 & \text { BA } 22\end{array}$

Table 3. Jackknife sensibility analysis. 


\begin{tabular}{|c|c|c|}
\hline All studies but... & $\begin{array}{l}\text { Right precentral gyrus } \\
(50,-10,40)\end{array}$ & $\begin{array}{l}\text { Left superior temporal gyrus } \\
(-58,-20,4)\end{array}$ \\
\hline Alonso 1 & Yes & Yes \\
\hline Alonso 2 & Yes & Yes \\
\hline Bai & Yes & Yes \\
\hline Ding & Yes & No \\
\hline Fryer & Yes & No \\
\hline Gao 1 & Yes & Yes \\
\hline Gao 2 & Yes & Yes \\
\hline Gao 3 & Yes & Yes \\
\hline Gou & Yes & Yes \\
\hline Guo 1 & Yes & Yes \\
\hline Guo 2 & Yes & Yes \\
\hline Guo3 & Yes & Yes \\
\hline Li 1 & Yes & Yes \\
\hline Li 2 & Yes & Yes \\
\hline Li 3 & Yes & Yes \\
\hline Liang 1 & Yes & Yes \\
\hline Liang 2 & Yes & Yes \\
\hline Liao & Yes & Yes \\
\hline Liu 1 & Yes & Yes \\
\hline Liu 2 & Yes & Yes \\
\hline Liu 3 & Yes & Yes \\
\hline Ren & Yes & Yes \\
\hline Turner & Yes & No \\
\hline Wang & Yes & Yes \\
\hline Yan & Yes & Yes \\
\hline Yu & Yes & Yes \\
\hline Zhao & Yes & Yes \\
\hline
\end{tabular}

Page 18/26 


\begin{tabular}{|lcc|}
\hline Zheng & Yes & Yes \\
Zhu & Yes & Yes \\
\hline
\end{tabular}

Yes: the region is reported if we extract the mentioned study of the analysis; No: the region is not reported if we extract the mentioned study of the analysis.

Table 4. Publication bias assessment.

\begin{tabular}{llll} 
Regions & $Z$ score & d.f & $p$ value \\
\hline Right precentral gyrus & -.67 & 27 & .504 \\
\hline Left superior temporal gyrus & -.22 & 27 & .751
\end{tabular}

$Z$ : contrast statistic of the publication bias, d.f: degree of freedom.

Table 5. Heterogeneity analysis of the positive and negative peaks.

\begin{tabular}{llllll} 
Peaks & $\tau$ & $Q$ & d.f & $p$ value & $P^{2}$ \\
\hline Positives & 0.051 & 44.805 & 21 & .002 & 50.89 \\
\hline Negatives & 0.071 & 70.336 & 26 & $<.001$ & 61.61
\end{tabular}

$\tau$ : variance of effect-size distribution, $Q$ : heterogeneity contrast statistic, $d$.f: degree of freedom, $P^{2}$ : rate of heterogeneity.

Table 6. Meta-regression of the possible mediator variables and effect sizes. 


\begin{tabular}{llc} 
Variables & Regression's coefficient $B_{i}$ & $p$ value \\
\hline Schizophrenia's sample size & -.008 & $<.001$ \\
\hline Control's sample size & -.007 & $<.001$ \\
\hline Schizophrenia's age & .007 & .419 \\
\hline Control's age & .008 & .385 \\
\hline Schizophrenia's gender & $<.001$ & .993 \\
\hline Control's gender & .002 & .755 \\
\hline PANSS total & .004 & .373 \\
\hline PANSS positive & -.005 & .754 \\
\hline PANSS negative & .016 & .316 \\
\hline PANSS general & $<.001$ & .979 \\
\hline Illness duration & .007 & .631 \\
\hline Quality of the studies & .214 & .272
\end{tabular}

Figures 


\section{Identification of studies via databases and registers}

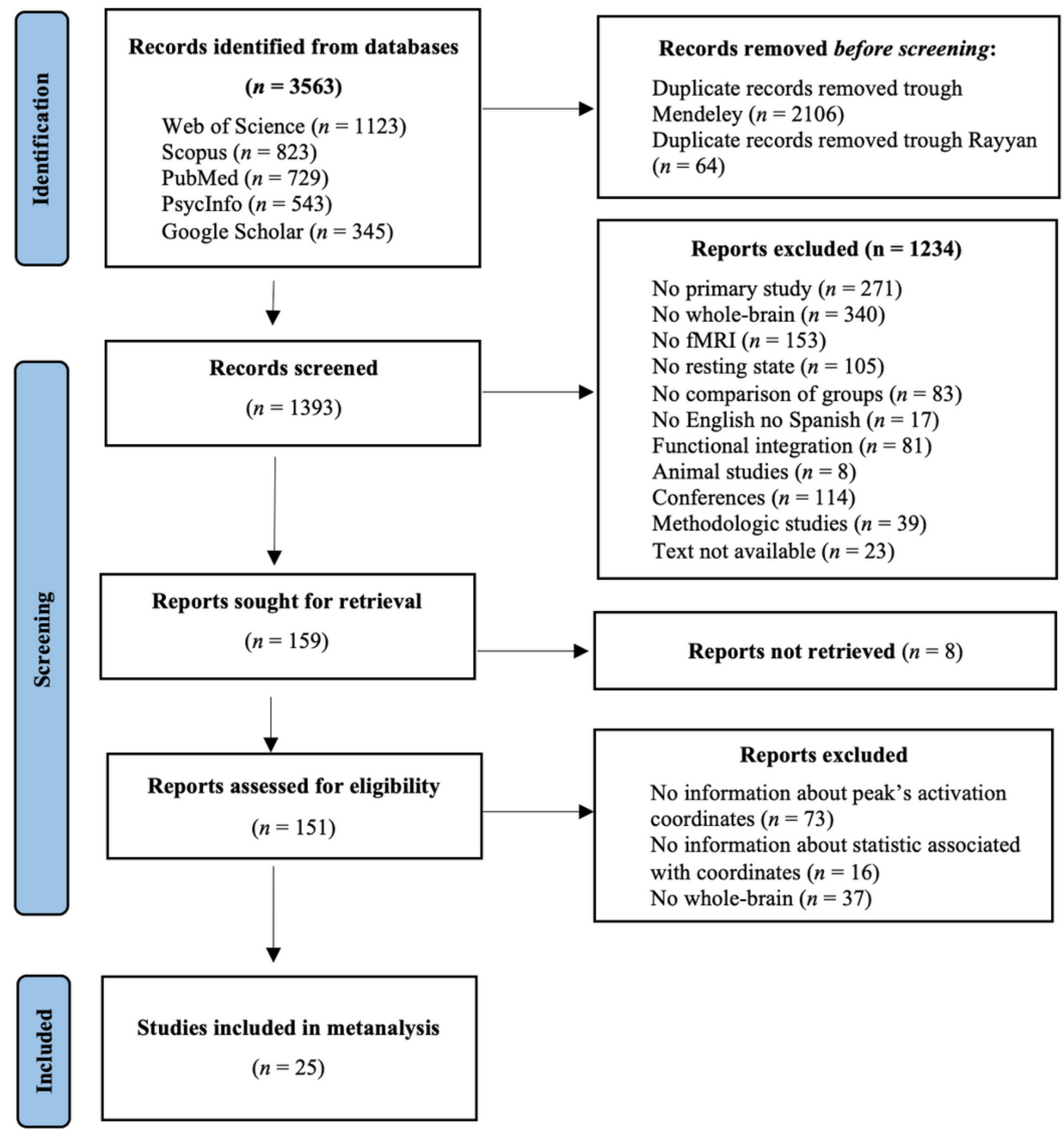

Figure 1

Flow chart of the metanalysis search conducted. 


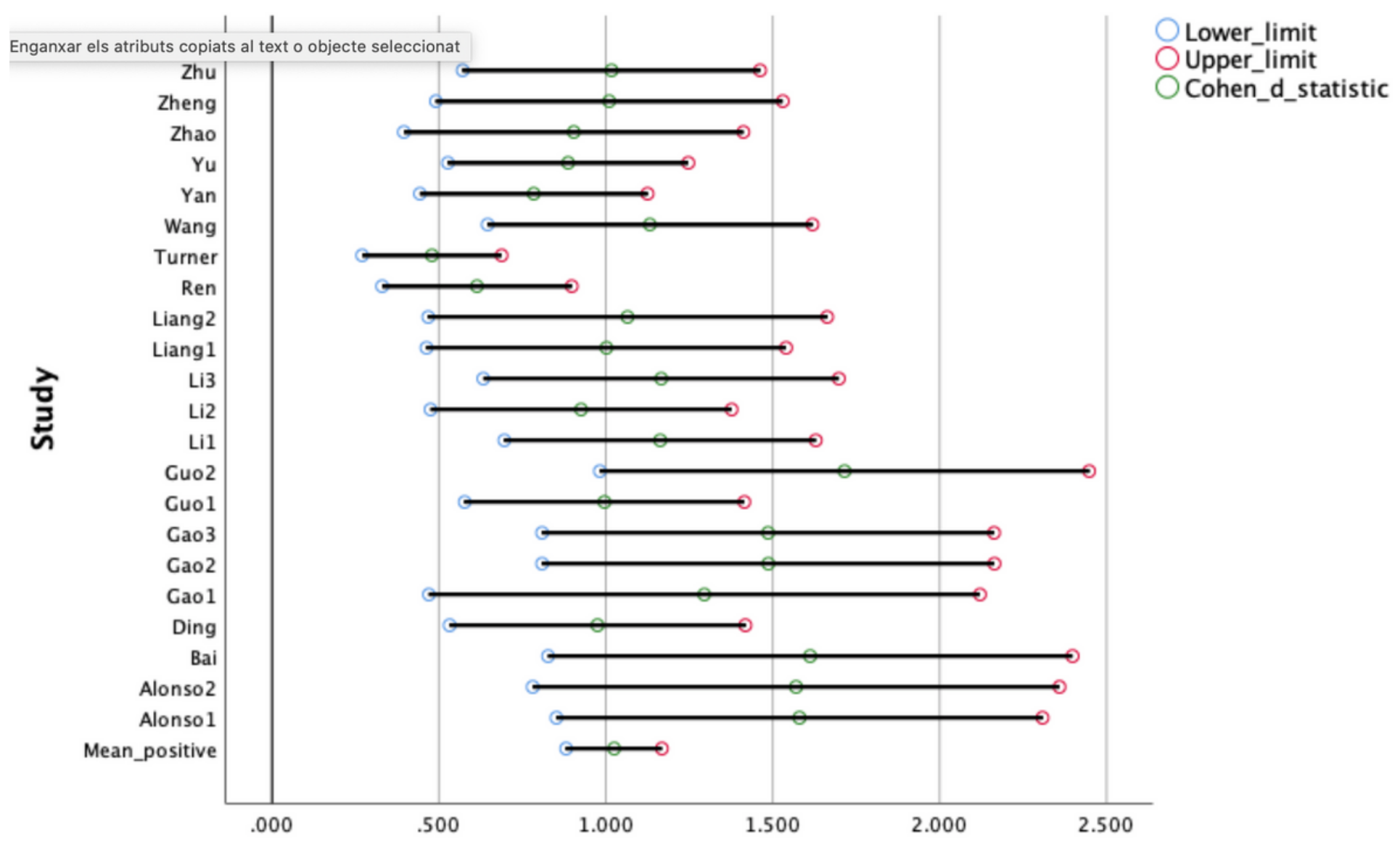

Cohen's d statistic

Figure 2

Forest Plot of the positive effect size (interval's confidence of 95\%) 


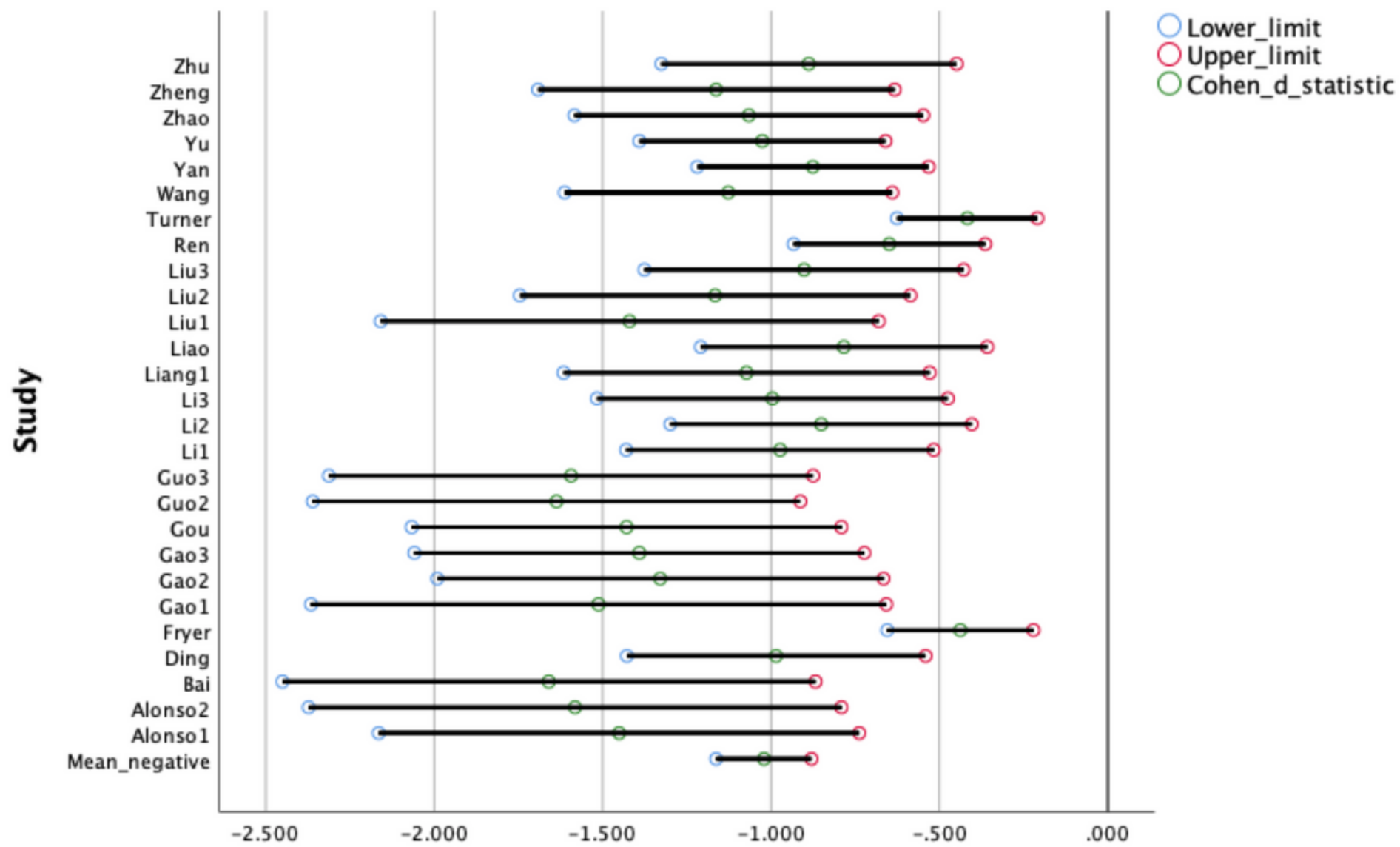

Cohen's d statistic

Figure 3

Forest Plot of the negative effect size (interval's confidence of 95\%) 

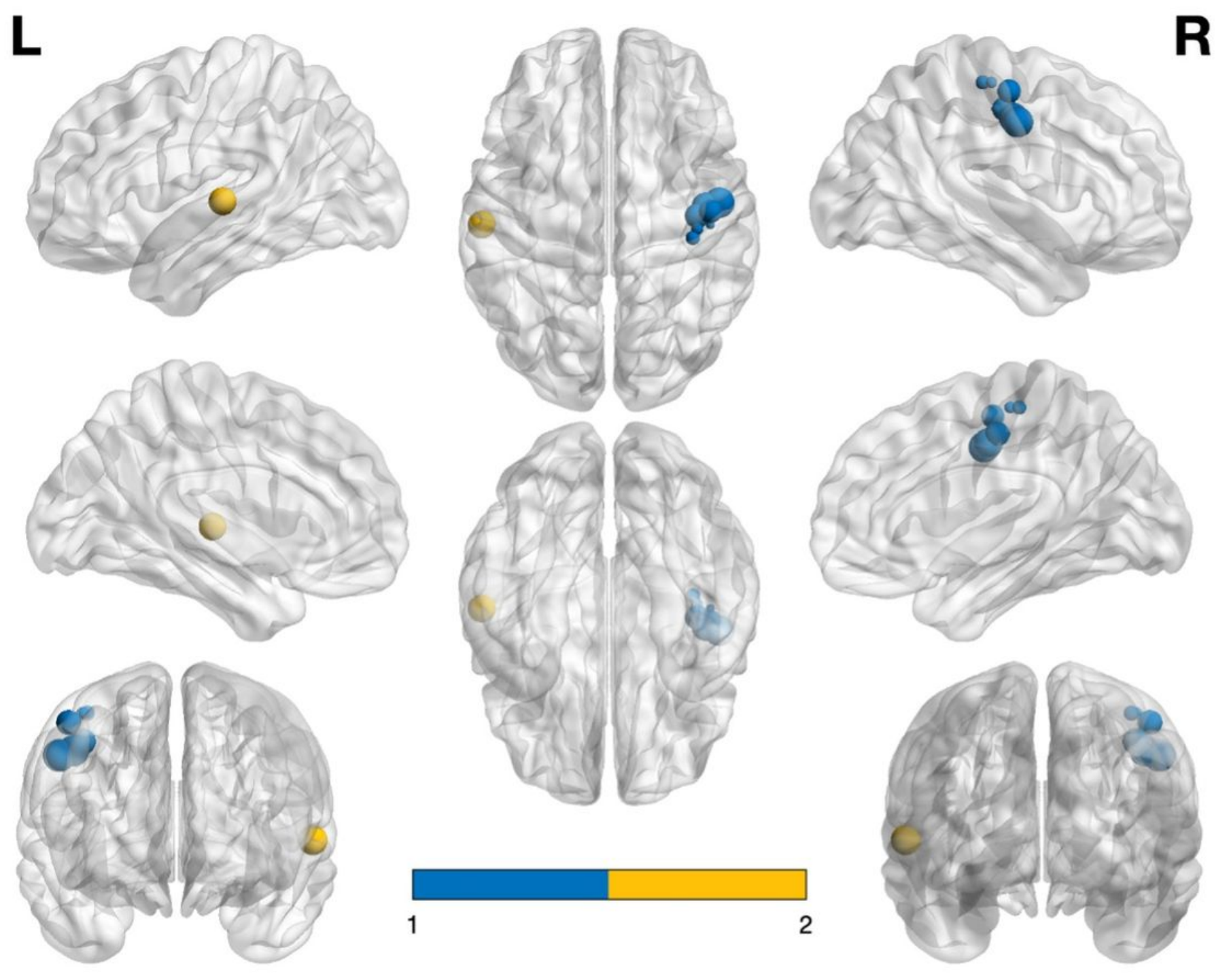

Figure 4

Representation of the most significant coordinates (in blue the right precentral gyrus and yellow the left superior temporal gyrus) that show hypoactivation in patients with schizophrenia compared to healthy controls 


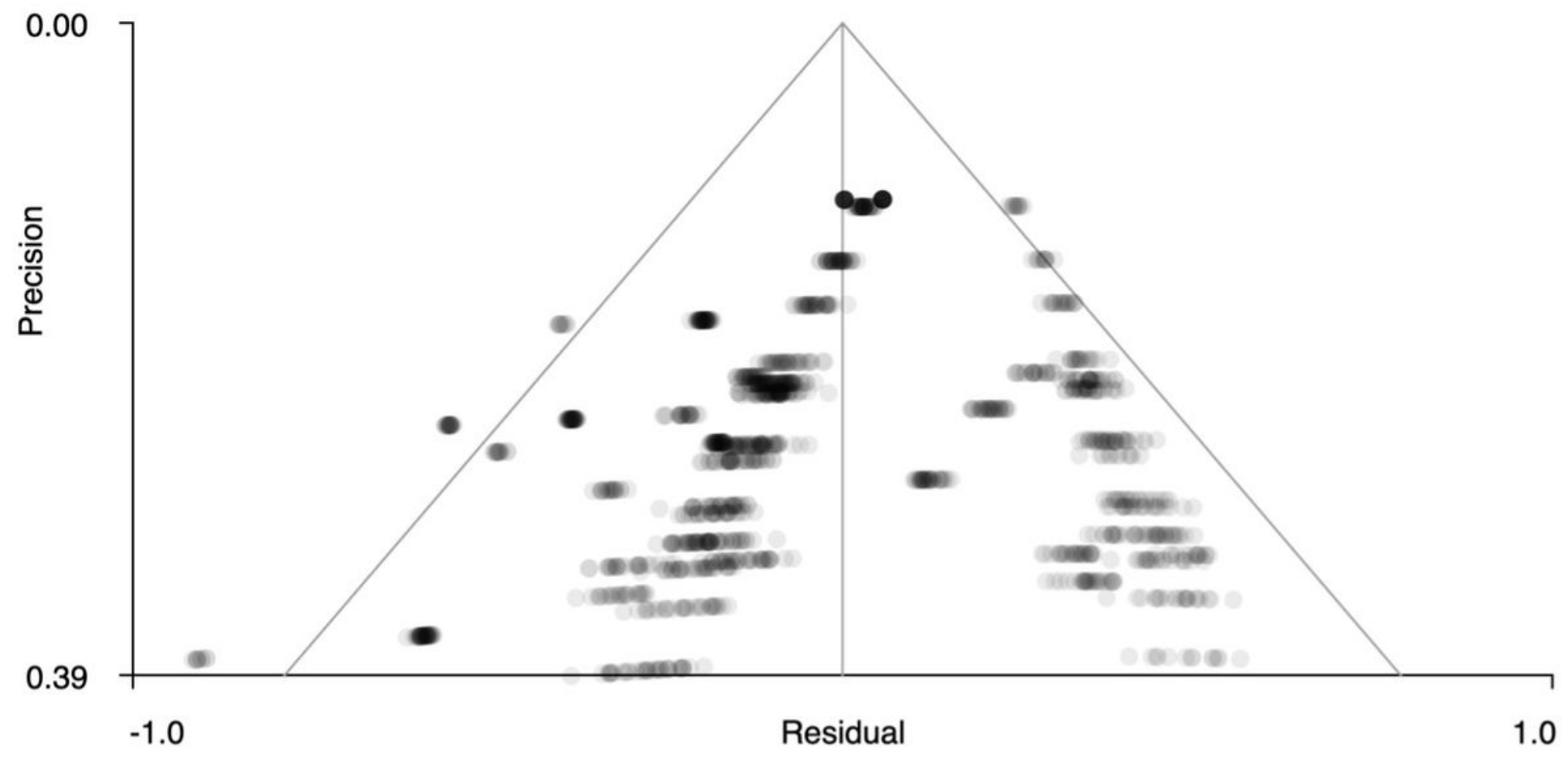

Figure 5

Funnel Plot of the effect associated with the coordinate of the right precentral gyrus

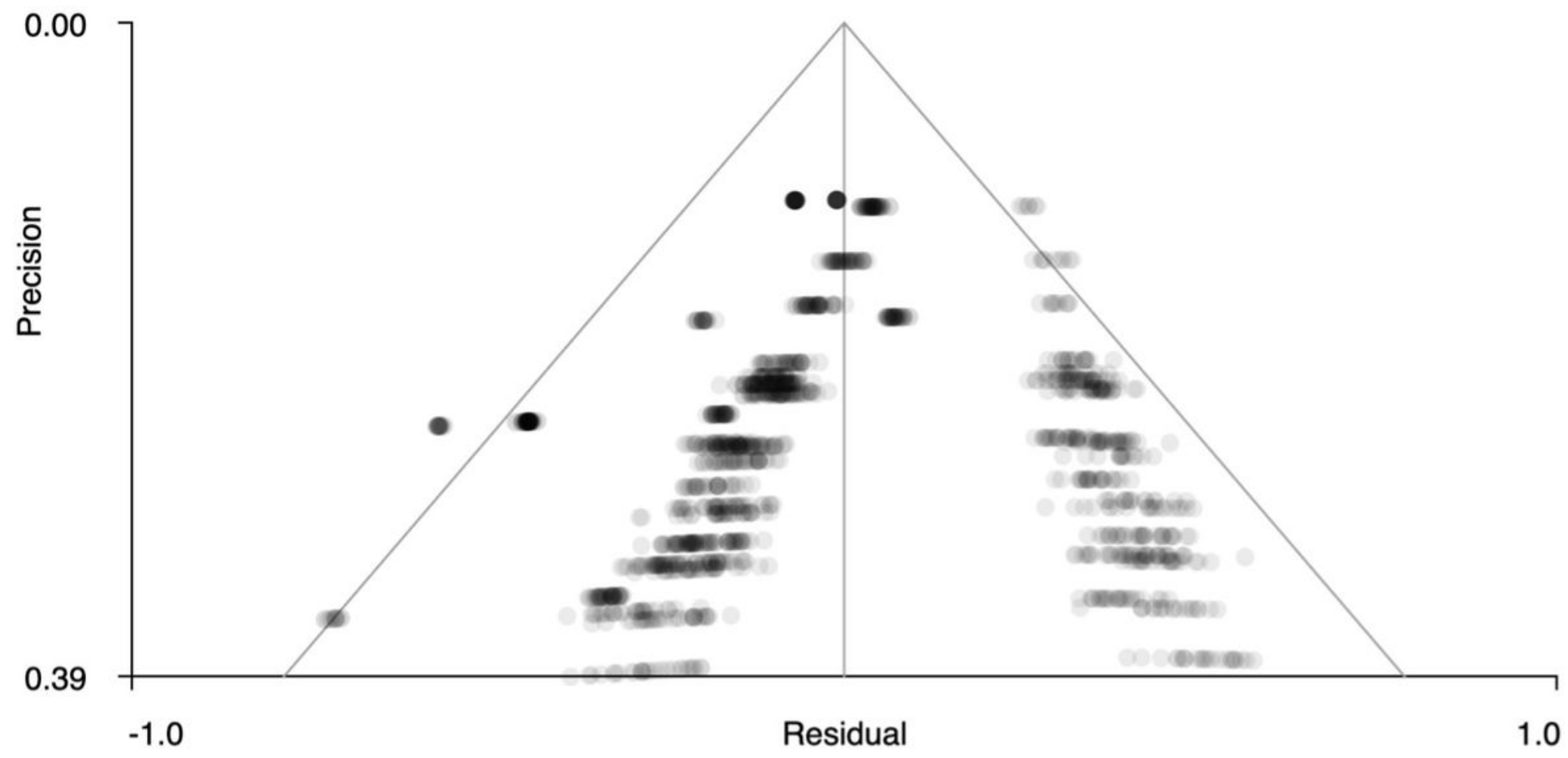

Figure 6

Funnel Plot of the effect associated with the coordinate of the left superior temporal gyrus 


\section{Supplementary Files}

This is a list of supplementary files associated with this preprint. Click to download.

- Appendix1.docx

- Appendix2.docx

- Appendix3.docx

- Appendix4.docx

- Appendix1.docx

- Appendix2.docx

- Appendix3.docx 